\title{
Treat and extend versus pro re nata regimens of ranibizumab and aflibercept in neovascular age-related macular degeneration: a comparative study
}

\author{
Michèle Augsburger ${ }^{1} \cdot$ Gian-Marco Sarra $^{2} \cdot$ Pascal Imesch $^{1}$ (D) \\ Received: 4 May 2019 /Revised: 19 June 2019 / Accepted: 21 June 2019 / Published online: 29 June 2019 \\ (C) The Author(s) 2019
}

\begin{abstract}
Purpose To compare treatment efficacy of anti-VEGF medications following pro re nata (PRN, "as needed", monthly injections only in case of active disease) or treat and extend (T\&E, progressive extension of treatment intervals up to 12 weeks depending on the clinical findings) treatment protocols in real-world conditions.

Methods Retrospective, observational study. Patients diagnosed with age-related macular degeneration and without pre-treatment undergoing routine anti-VEGF treatment in one eye clinic in Switzerland were included. Treatment was performed according to local practices, using ranibizumab or aflibercept, and following T\&E or PRN regimens. Changes in logMAR and injection intervals (time between two injections) for specific treatment periods were evaluated descriptively and using mixed models.

Results In total, 1071 patients with 1332 treated eyes (ranibizumab/PRN 722, ranibizumab/T\&E 191, aflibercept/T\&E 419) were included in the analyses. At baseline, $\operatorname{logMAR}($ mean $\pm \mathrm{SD}$ ) was similar in both ranibizumab treatment groups (PRN $0.63 \pm 0.43$, $\mathrm{T} \& \mathrm{E} 0.57 \pm 0.42$ ). In the ranibizumab/PRN group, logMAR was about 0.1 lower for all time intervals in the initial and maintenance phases in comparison with baseline, indicating an improvement in VA. By comparison, logMAR improved more strongly in the ranibizumab/T\&E group (16 to <22 months, -0.19 [ $-0.23-0.15]$ ) in comparison with baseline. Comparing ranibizumab/T\&E vs. aflibercept/T\&E groups, improvements in logMAR were similar over time. In the maintenance phase, the rate of patients with a clinically relevant improvement in visual acuity ( $>0.2 \log$ MAR) was higher in both T\&E groups compared with the ranibizumab/PRN group. Injection intervals in the maintenance phase in ranibizumab/T\&E group gradually expanded over time; whereas in the aflibercept/T\&E group, injection intervals remained relatively stable.

Conclusions Ranibizumab used according to T\&E protocol yielded a stronger improvement in logMAR, compared with PRN protocol with longer injection intervals than aflibercept/T\&E. This large real-world data assessment supports previous data on the superiority of T\&E treatment.
\end{abstract}

Keywords Age-related macular degeneration (AMD) - Pro re nata (PRN) - Treat and extend (T\&E) - Anti-VEGF treatment · Aflibercept · Ranibizumab

\section{Introduction}

Age-related macular degeneration (AMD) is a common, chronic retinal disease that may cause dramatic impairment of visual acuity (VA). Neovascular AMD (nvAMD), a subtype, may be associated with the development of defunct

Pascal Imesch

pascal@imesch.com

1 EYEPARC eye clinic, Spitalgasse 26, 3011 Bern, Switzerland

2 Augenpraxis Emme, Burgdorf, Switzerland macular blood vessels. NvAMD is characterized by leakage of fluid or blood into the macula, which severely affects central VA and may lead to legal blindness. Recent years have seen dramatic improvements in the therapeutic options of nvAMD, as anti-vascular endothelial growth factors (antiVEGF), applied by intravitreal injection, inhibit development of these novel, aberrant blood vessels and the ensuing macular fluid leakage successfully.

Anti-VEGF treatment options have upended treatment of several retinal diseases, yet most notably of nvAMD, where treatment has to be applied often indefinitely and in short time intervals. Thus, disease management, albeit highly effective, constitutes a high burden for patients and healthcare providers 
alike. Moreover, every single injection bears a small yet significant risk of endophthalmitis, a feared complication threatening eyesight, making any attempt of reducing injection frequency a crucial, patient-relevant objective. [1]

Consequently, there is still some uncertainty on the optimal treatment regimen that manages to balance all pros and cons optimally. Importantly, results of the pivotal phase III trials on the use of ranibizumab and aflibercept were based on regular, uninterrupted dosing protocols. [2-5] As a first attempt to alleviate high treatment burden, the pro re nata (PRN) regimen was introduced, a treatment protocol where follow-up intervals remain fixed, while decisions to carry out an injection are based on the anatomic findings at each respective visit. [6-8] Notably, by following such a PRN treatment protocol, injections are based on prior pathological OCT findings and, therefore, fall short of preventing the damaging yo-yo effect of retinal fluid accumulation. In consequence, functional PRN results fared worse as compared with the pivotal phase III trials; in particular, initial VA gains could not be maintained as well during a longer follow-up time. [7-9] In order to develop a protocol with a reduced treatment burden, while at the same time ensuring timely treatment before any macular fluid accumulation occurs, another treatment regimen, the treat and extend (T\&E) protocol, was developed. In T\&E regimens, the time interval of follow-up visits will be adjusted based on the patient's clinical course - i.e. if a patient shows no sign of an active disease (e.g. the macula remains dry, without any leakage), intervals will be extended; if there is fluid accumulation, the next interval will be shortened. Importantly, at each patient's visit following T\&E, an injection will be performed; the current clinical status only has an impact on the duration of the next injection interval. Therefore, T\&E may also be called "pro-active" in contrast to the more "reactive" PRN protocol. Prior studies have shown efficacy of T\&E, but are mostly still limited to smaller studies. [10-15] As pointed out by the American Academy of Ophthalmology, larger studies on the efficacy of T\&E regimens are clearly warranted. [16]

The primary aim of this study was therefore to compare efficacy of PRN vs. T\&E treatment protocols using ranibizumab, as well as comparing the effect of ranibizumab and aflibercept in clinical practice using the T\&E protocol, both with regard to visual acuity (evaluated using logMAR i.e. the logarithm of the minimum angle of resolution) and with regard to the injection frequency.

\section{Patients and methods}

\section{Study design, patient selection and treatment intervention}

This was a retrospective comparative study. Patients diagnosed with nvAMD and without any prior anti-VEGF pre- treatment or photodynamic therapy (PDT) were included consecutively in the study. Participation did not affect any prior or current treatment decisions. All patients underwent standard baseline diagnostic procedures including optical coherence tomography (OCT) and fluorescein angiography (FA). All procedures including follow-up visits took place at the Eyeparc clinic in Bern, Switzerland. Anti-VEGF treatment consisted of ranibizumab (Lucentis ${ }^{\circledR}$ ) and aflibercept (Eylea®). Before December 1, 2012, aflibercept was not available in Switzerland; the routine treatment regimen for all patients was ranibizumab according to PRN. PRN treatment was initiated with three monthly injections. Decisions for subsequent injections were based on monthly OCT findings indicative of active disease (e.g. intraretinal/subretinal leakage or haemorrhage).

After December 1, 2012, treatment regimen for all patients was switched to T\&E using either ranibizumab or the newly introduced aflibercept according to the physician's discretion. During T\&E regimen, initial injection interval was 4 weeks between the first and second injection, and was then extended by 2 weeks to a maximum of 12 weeks in case of an optimal result and a stable, non-active disease (e.g. no intraretinal/ subretinal leakage or haemorrhage) based on OCT and fundoscopy findings. Similarly, upon recurring signs of active disease, the injection intervals were respectively shortened by 2 weeks (to a minimum injection interval of 4 weeks). For analysis, only the first treatment scheme of each treated eye was considered.

All research followed the tenets of the Declaration of Helsinki, and the study was approved by an ethics committee in Switzerland (swissethics).

\section{Statistics and mathematical analyses}

Descriptive statistics were performed for patient and baseline characteristics for each of the three analysis groups (ranibizumab PRN, ranibizumab T\&E, aflibercept T\&E). For all descriptive analyses, "treated eyes" were considered as the statistical unit, since the treatment period as well as the treatment scheme and/or medication could differ between the two eyes of one patient.

As VA was measured on a decimal scale (Snellen), it was for the purpose of this analysis transformed to logMAR using the following established formula: $\log \mathrm{MAR}=\log 10(1 / \mathrm{VA})$. The outcomes of interest were logMAR and injection intervals (time between two injections). In the following analyses, $\log$ MAR was compared for different time intervals. To this effect, 2-month intervals were created for the initial phase of four months i.e. the "loading phase"; and 6-month intervals were created for the subsequent maintenance phase. A "spatial" covariance structure was used. The elements of this covariance matrix decline with increasing time between two injections i.e. the larger the time 
Table 1 Patient age at treatment initiation, duration of treatment, numbers of examination, and numbers of injections in all three treatment groups. $N$ number of examined eyes, $P R N$ pro re nata, $S D$ standard deviation, $T \& E$ treat and extend)

\begin{tabular}{lllllll}
\hline & Group & N & Mean & SD & Median & Range \\
\hline Age (years) & Ranibizumab PRN & 722 & 77.9 & 8.9 & 79.5 & $34.0-96.0$ \\
& Ranibizumab T\&E & 191 & 79.8 & 8.7 & 81.0 & $48.0-97.0$ \\
& Aflibercept T\&E & 419 & 79.8 & 7.6 & 81.0 & $58.0-96.0$ \\
Duration of treatment (in months) & Ranibizumab PRN & 722 & 13.5 & 16.2 & 6.9 & $0.0-75.8$ \\
& Ranibizumab T\&E & 191 & 15.7 & 14.6 & 12.0 & $0.0-53.1$ \\
& Aflibercept T\&E & 419 & 15.6 & 13.1 & 12.7 & $0.0-54.0$ \\
Number of examinations & Ranibizumab PRN & 722 & 11.1 & 14.8 & 5.0 & $1.0-95.0$ \\
& Ranibizumab T\&E & 191 & 9.4 & 7.3 & 8.0 & $1.0-39.0$ \\
& Aflibercept T\&E & 419 & 10.3 & 8.1 & 9.0 & $1.0-43.0$ \\
Number of injections & Ranibizumab PRN & 722 & 6.3 & 6.1 & 4.0 & $2.0-36.0$ \\
& Ranibizumab T\&E & 191 & 9.4 & 7.3 & 8.0 & $4.0-39.0$ \\
& Aflibercept T\&E & 419 & 10.3 & 8.1 & 9.0 & $1.0-43.0$ \\
\hline
\end{tabular}

between two injections the lower the correlation between the two respective $\log$ MAR values.

To validate the extent of changes in $\log$ MAR over time, the differences between eye-specific $\log$ MAR averages and baseline values were calculated for each time interval. An absolute difference of $<0.2$ was considered as no clinically relevant change, a difference $\geq 0.2$ as a worsening and a difference $\leq 0.2$ as an improvement in eyesight. A change in $\log$ MAR by 0.2 is equivalent to a two-line change on the Snellen chart.

For analysis of injection intervals, two time frames were distinguished, the loading phase ( $<4$ months after first injection) and the maintenance phase ( $\geq 4$ months after first injection). Additionally, the analyses for injection intervals were performed for yearly intervals within the maintenance phase. Injection intervals overlapping two treatment intervals were excluded. Mixed models were used to evaluate how injection intervals changed over time (complete maintenance phase as well as yearly treatment periods) in comparison with the loading phase.
Results are shown from the initial phase and from the first 3 years of the maintenance phase $(<52$ months); after 52 months, the number of treated eyes were too low for statistically valid interpretation.

In addition, subgroup analyses were performed, analysing subgroups stratified by age and gender.

All statistical analyses were performed with SAS ${ }^{\circledR}$ package (version 9.4).

\section{Results}

\section{Patient demographics}

From August 2006 to August 2017, overall, 1071 nvAMD patients (1332 eyes) undergoing initial anti-VEGF treatment at the Eyeparc clinic were analysed. In total, patients received 14,130 anti-VEGF injections according to local routine practice.
Fig. 1 Changes in logMAR (together with 95\% CI) for each time interval in comparison with baseline in patients using ranibizumab according to $\mathrm{PRN}$ or $\mathrm{T} \& \mathrm{E}$ treatment protocol. The number of examinations in each time period and treatment group is given next to the confidence intervals. (Legend: CI, confidence interval; mth, month; PRN, pro re nata; T\&E, treat and extend)

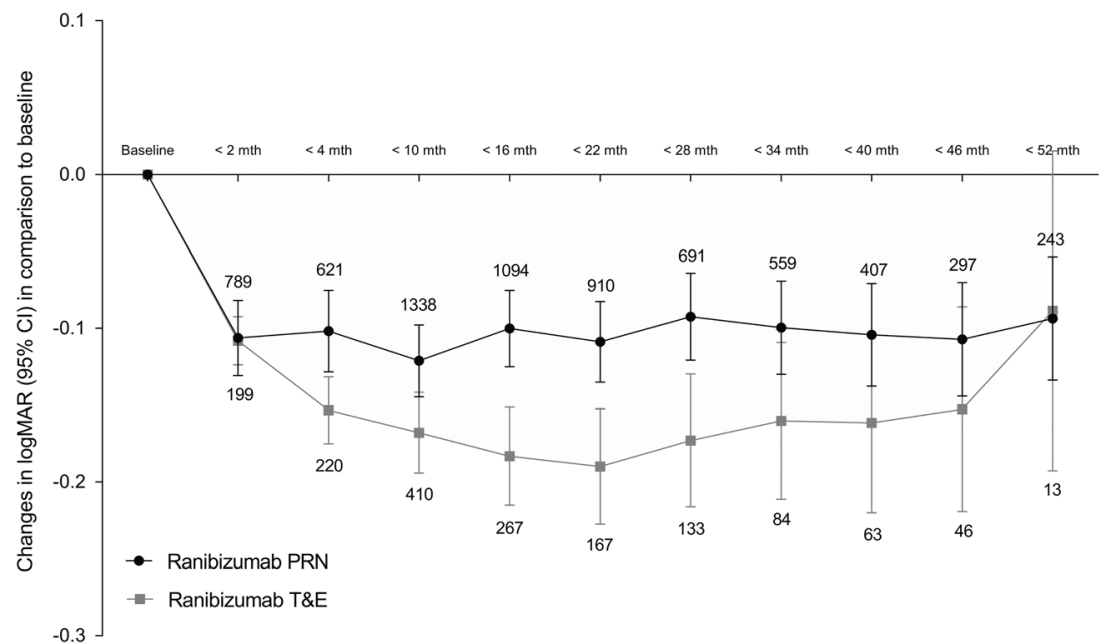


Patient characteristics were largely similar in all three treatment groups; mean patient age was just below 80 years (Table 1). Across all treatment groups, there was a considerable preponderance of female patients (ranibizumab PRN 71.2\%, ranibizumab T\&E $69.1 \%$, aflibercept T\&E 66.1\%). Duration of treatment and numbers of injections/examinations are shown in Table 1.

In addition, mean numbers of annual examinations and annual injections were calculated, including only patients who received treatment for at least 12 months. The number of injections per year (which in the T\&E groups equals the number of examinations) was highest in the aflibercept T\&E group $(7.5 ; n=203)$, followed by ranibizumab T\&E $(6.8 ; n=$ 96) and PRN $(5.0 ; n=445)$. In the PRN group, patients had the highest number of annual examinations, on average 9.2 per year $(n=277)$; accordingly, patients received an injection at approximately every other examination.

\section{Comparison of PRN vs. T\&E using ranibizumab}

At baseline, logMAR (mean $\pm \mathrm{SD}$ ) was similar in both ranibizumab treatment groups $(\mathrm{PRN} 0.63 \pm 0.43$, TE $0.57 \pm$ 0.42 ). In the loading phase, logMAR values were about 0.1 lower compared with baseline reflecting an improvement in VA in patients treated according to the PRN scheme (Fig. 1). This improvement remained stable during the maintenance phase. In patients of the T\&E group, a similar improvement in logMAR was detected in the first time interval of the loading phase ( 1 day-<2 months after baseline, -0.11 [95\% CI $0.12,-0.09])$. However, $\log$ MAR further decreased in comparison with baseline also during the loading and maintenance phase. The strongest decrease was observed after $16-<$ 22 months $(-0.19[-0.23,-0.15])$.

After the first injection, VA remained unchanged in $70.4 \%$ and improved in $25.0 \%$ of the patients in the ranibizumab/ PRN treatment group (Table 2). For all time intervals in the maintenance phase, about $50 \%$ of the patients showed an unchanged logMAR compared with baseline. However, the proportion of patients with an improvement decreased (e.g. 4 to $<$ 10 months $36.1 \%$ vs. 46 to $<52$ months $12.5 \%$ ), and the proportion of patients with a worsened logMAR increased (e.g. 4 to $<10$ months $11.5 \%$ vs. 46 to $<52$ months $37.5 \%$ ) over time. In contrast, during the treatment period, the frequency of ranibizumab/T\&E patients with a worsening in VA remained low $(<10 \%$, except for time interval 46 to $<$ 52 months), and the frequency of patients with an improvement gradually increased from $28.3 \%$ ( 1 day to $<2$ months) to at most $57.1 \%$ (40 to $<46$ months).

\section{Comparison of ranibizumab vs. aflibercept following T\&E treatment protocol}

We observed similar mean $\log$ MAR values and standard deviations for patients in both $\mathrm{T} \& \mathrm{E}$ groups at baseline

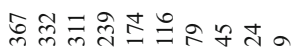

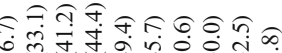

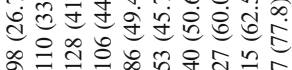
(1)

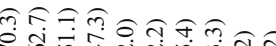

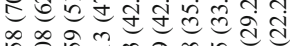

อิกำดวิ

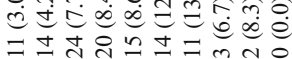
$=-\mathrm{N}-\neg-m \mathrm{n}$

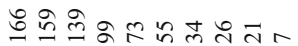

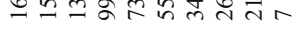

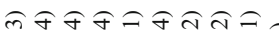

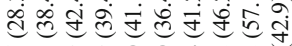

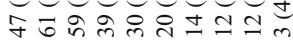

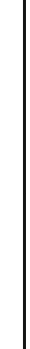

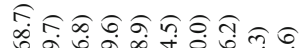

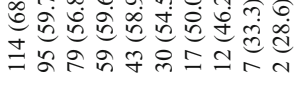

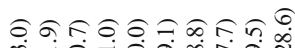

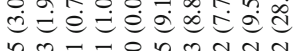

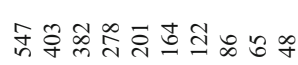

(5)

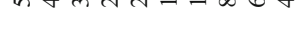

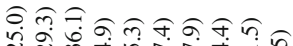

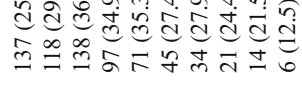

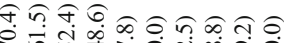

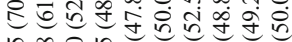

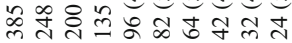

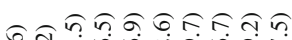

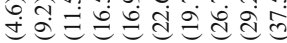

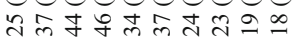

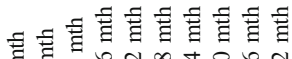

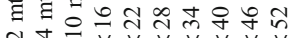

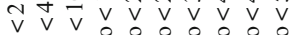
o 0 \%

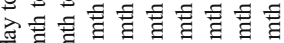

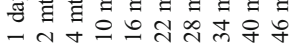


Fig. 2 Changes in $\log M A R$ (together with 95\% CI) in comparison with baseline for each time period in patients using ranibizumab or aflibercept following T\&E treatment protocol. The number of examinations in each time period and treatment group is given next to the confidence intervals.

(Legend: CI, confidence interval; mth, month; PRN, pro re nata;

$\mathrm{T} \& \mathrm{E}$, treat and extend)

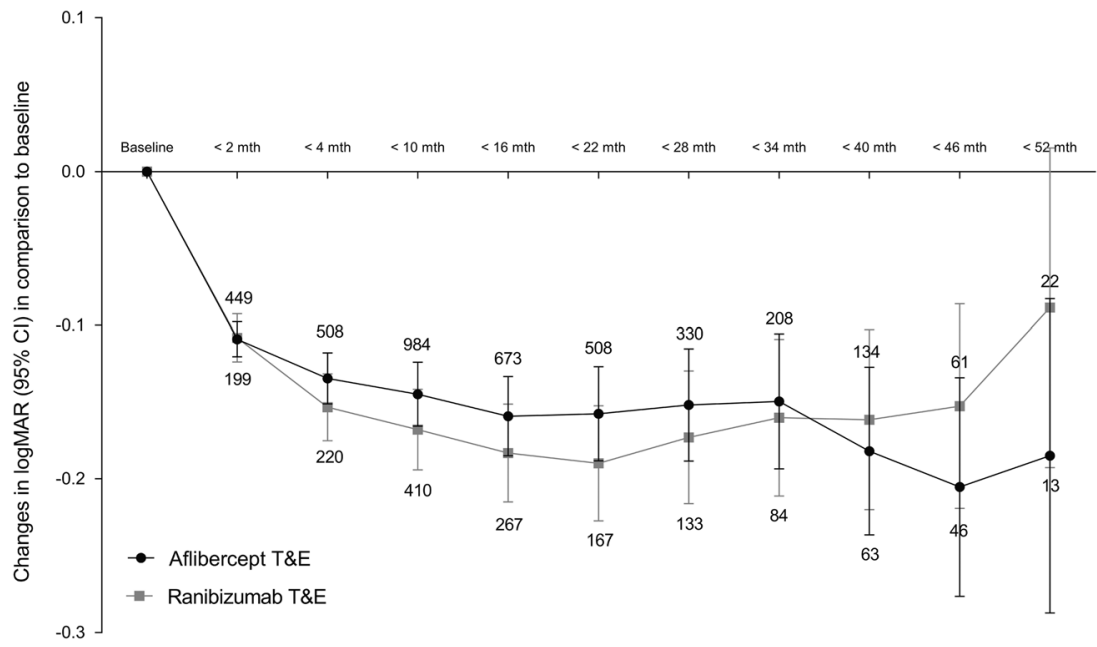

(ranibizumab $0.57 \pm 0.42$, aflibercept $0.60 \pm 0.46$ ). The changes in $\log$ MAR in the loading and maintenance phase in comparison with baseline were similar in both treatment groups (Fig. 2). Based on the analyses using the categorized differences in $\log$ MAR, we observed a higher proportion of patients with an improvement in VA in the maintenance phase in patients treated with aflibercept (e.g. 34 to $<40$ months, $60 \%$ ) compared with ranibizumab (e.g. 34 to $<40$ months, $46.2 \%$ ) (Table 2). However, also the proportion of patients with a VA worsening tended to be somewhat higher in patients using aflibercept for almost all time intervals.

\section{Comparison of T\&E injection intervals}

Injection intervals in the initial phase were similar between the $\mathrm{T} \& \mathrm{E}$ groups, as expected (ranibizumab 36.6 days $[35.6,37.6]$, aflibercept 34.9 days $[34.2,35.5])$. In both treatment groups, injection intervals were longer in the maintenance phase in comparison with the loading phase (ranibizumab 29.3 [25.0, 33.6] days longer compared with the loading phase, aflibercept 23.6 days $[21.2,26.1])$.
In the first year of the maintenance phase ( 4 months to $<$ 16 months), similar time increments between two injections in comparison with the loading phase were observed in both treatment groups (aflibercept 20.2 days [18.1, 22.3], ranibizumab 22.7 days $[19.5,25.9]$ ) (Fig. 3). While injection intervals did not change essentially in the following treatment years in patients treated with aflibercept, injection intervals markedly increased in patients treated with ranibizumab. However, these changes should be interpreted cautiously as the number of included time intervals decreases considerably over time.

\section{Subgroup analysis}

In the subgroup analysis, patients younger than the median age of the study population (80 years) treated according $\mathrm{T} \& \mathrm{E}$ with either ranibizumab or aflibercept experienced a stronger increase in VA, yet also required shorter injection intervals than older patients. No notable gender-specific differences were detected (data not shown).
Fig. 3 Changes in injection intervals in days (together with $95 \% \mathrm{CI}$ ) in comparison with the loading phase for different yearly intervals of the maintenance phase. The number of injection intervals in each time period and treatment group is given next to the confidence intervals. (Legend: $\mathrm{CI}$, confidence interval; $\mathrm{mth}$, month; PRN, pro re nata; T\&E, treat and extend)

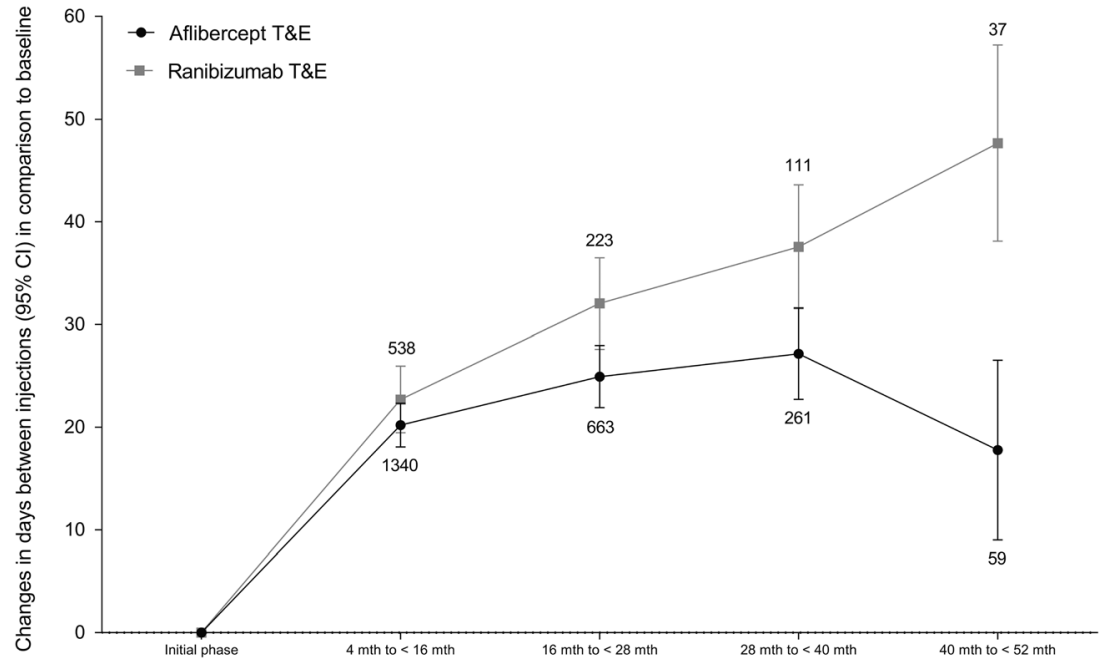




\section{Discussion}

Despite ongoing efforts to determine optimal treatment regimens of anti-VEGF treatment, a gold standard has not been determined yet, and the trade-off between treatment efficacy versus patient burden and injection complications remains controversial.

The results of this study largely confirmed available data of the published literature $[10-15,17]$. Notably, all three treatment regimens yielded favourable logMAR stabilization over a substantial observational phase of more than 40 months, and the initial gains of logMAR were for the most part maintained. The T\&E treatment protocol proved to be overall more favourable in terms of speed and strength of $\log$ MAR improvement. As expected, annual numbers of examinations were higher in the PRN group than in the two T\&E groups. However, yearly numbers of injections were lowest in the PRN group. This may be partly due to the more rigid and standardized injection scheme in the T\&E regimen. Another reason may be better patient compliance in light of the reduced patient burden in the T\&E groups. According to the authors' experience, patients treated according to T\&E appeared to be less anxious during the visit, knowing in advance that they would definitely receive an injection. Patients also expressed their appreciation for the prolonged intervals between consultations. As no ancillary data were collected on the patients' subjective experience, no statistical evaluation could be performed to further support this observation.

Interestingly, ranibizumab and aflibercept T\&E injection interval duration, while initially of similar length, started to diverge 2 years after the loading phase. We observed longer injection intervals on average in patients treated with ranibizumab than with aflibercept 3 years after the loading phase. These results are surprising, as aflibercept can be dosed less frequently than ranibizumab (every 8 weeks after loading phase) according to the Summary of Product Characteristics (SmPC). In fact, less frequent treatment was seen as a key advantage of aflibercept in comparison with other drugs. Further studies on the possible advantage of ranibizumab over aflibercept with respect to frequency of treatment will be therefore coveted, as any reduction in injections is highly relevant for patients, healthcare providers and national healthcare systems alike.

A strength of this study is certainly its high numbers of examined eyes and injections, along with its comparative results not only on different treatment protocols (T\&E vs. PRN), but also on different drugs. To exclude effects of prior treatment, only patients receiving initial anti-VEGF injections were included. Thus, all data after a treatment change were excluded. The observational, retrospective design of this study implicates, naturally, certain limitations of the data quality, and a lower evidence level than those from a clinical trial. Despite the consecutive patient recruitment, implicit selection bias cannot be ruled out entirely. Moreover, treating physicians displayed changing preferences for ranibizumab vs. aflibercept over time: initially, they tended to choose ranibizumab for intraretinal leakage i.e. for somewhat milder cases, and aflibercept for patients with more severe findings, including subretinal leakage and signs of subretinal fibrosis. In a second phase, physicians treated all patients with aflibercept. In the last, third phase, physicians attempted to choose between both substances randomly, albeit in a subjective way. Thus, a certain degree of selection bias must be taken into account. In summary, these changing preferences may be the cause for the differing size of the patient groups, and it suggests that the aflibercept patients tended to be the more severe cases. Notwithstanding, the initial VAs in the ranibizumab $\mathrm{T} \& \mathrm{E}$ and aflibercept $\mathrm{T} \& \mathrm{E}$ groups were comparable, which again may relativize that assumption. However, as other previous publications described a higher efficacy of aflibercept than ranibizumab, this potential weakness of this study's design must be taken into consideration.

Overall, the differences in mean VA values between all treatment groups were relatively small. However, our results showed clear trends supporting the prevailing hypothesis of T\&E being superior to PRN treatment. Notably, in the PRN group, there was a considerable rate of patients who experienced a worsening of logMAR $(>0.2$, equivalent to a 2-line change on the Snellen chart); whereas in the T\&E groups, $\log$ MAR worsened only in very few patients.

In summary, this large observational study confirms prior data on the efficacy of anti-VEGF treatment, provides additional data on the potential superiority of T\&E over PRN protocols, and raises the hypothesis of ranibizumab warranting fewer injections than aflibercept on the long run. Further studies will be required to further elucidate these findings.

Acknowledgements GKM Gesellschaft für Therapieforschung mbH provided support in completion of this manuscript.

\section{Compliance with ethical standards}

Conflict of interest PI has received research grants from Novartis and Bayer; GS has received a speaker honorarium from Bayer; MA declares that she has no conflict of interest.

Ethical approval All procedures performed in studies involving human participants were in accordance with the ethical standards of the swissethics committee in Berne (ID 2016-00650) and with the 1964 Helsinki declaration and its later amendments or comparable ethical standards.

Informed consent Due to the retrospective study design, and because several patients had already died at the start of the study, informed consent could not be obtained in all cases. Thus, the ethics committee allowed the use of anonymized data without obtaining prior informed consent for this study. 
Open Access This article is distributed under the terms of the Creative Commons Attribution 4.0 International License (http:// creativecommons.org/licenses/by/4.0/), which permits unrestricted use, distribution, and reproduction in any medium, provided you give appropriate credit to the original author(s) and the source, provide a link to the Creative Commons license, and indicate if changes were made.

\section{References}

1. Pilli S, Kotsolis A, Spaide RF, Slakter J, Freund KB, Sorenson J, Klancnik J, Cooney M (2008) Endophthalmitis associated with intravitreal anti-vascular endothelial growth factor therapy injections in an office setting. Am J Ophthalmol 145(5):879-882

2. Brown DM, Kaiser PK, Michels M, Soubrane G, Heier JS, Kim RY, Sy JP, Schneider S (2006) Ranibizumab versus verteporfin for neovascular age-related macular degeneration. N Engl J Med 355(14):1432-1444

3. Heier JS, Brown DM, Chong V, Korobelnik J-F, Kaiser PK, Nguyen QD, Kirchhof B, Ho A, Ogura Y, Yancopoulos GD (2012) Intravitreal aflibercept (VEGF trap-eye) in wet age-related macular degeneration. Ophthalmology 119(12):2537-2548

4. Rosenfeld PJ, Heier JS, Hantsbarger G, Shams N (2006) Tolerability and efficacy of multiple escalating doses of ranibizumab (Lucentis) for neovascular age-related macular degeneration. Ophthalmology 113(4):623-632 e621

5. Mitchell P (2011) A systematic review of the efficacy and safety outcomes of anti-VEGF agents used for treating neovascular agerelated macular degeneration: comparison of ranibizumab and bevacizumab. Curr Med Res Opin 27(7):1465-1475

6. Group CR (2011) Ranibizumab and bevacizumab for neovascular agerelated macular degeneration. N Engl J Med 364(20):1897-1908

7. Maguire MG, Martin DF, Ying G-S, Jaffe GJ, Daniel E, Grunwald JE, Toth CA, Ferris FL III, Fine SL, Group CoA-rMDTTR (2016) Five-year outcomes with anti-vascular endothelial growth factor treatment of neovascular age-related macular degeneration: the comparison of age-related macular degeneration treatments trials. Ophthalmology 123(8):1751-1761

8. Lalwani GA, Rosenfeld PJ, Fung AE, Dubovy SR, Michels S, Feuer W, Davis JL, Flynn HW Jr, Esquiabro M (2009) A variable-dosing regimen with intravitreal ranibizumab for neovascular age-related macular degeneration: year 2 of the PrONTO Study. Am J Ophthalmol 148(1):43-58 e41

9. Holz FG, Schmitz-Valckenberg S, Fleckenstein M (2014) Recent developments in the treatment of age-related macular degeneration. J Clin Invest 124(4):1430-1438

10. Shienbaum G, Gupta OP, Fecarotta C, Patel AH, Kaiser RS, Regillo CD (2012) Bevacizumab for neovascular age-related macular degeneration using a treat-and-extend regimen: clinical and economic impact. Am J Ophthalmol 153(3):468-473 e461

11. Gupta OP, Shienbaum G, Patel AH, Fecarotta C, Kaiser RS, Regillo CD (2010) A treat and extend regimen using ranibizumab for neovascular age-related macular degeneration: clinical and economic impact. Ophthalmology 117(11):2134-2140

12. Oubraham H, Cohen SY, Samimi S, Marotte D, Bouzaher I, Bonicel P, Fajnkuchen F, Tadayoni R (2011) Inject and extend dosing versus dosing as needed: a comparative retrospective study of ranibizumab in exudative age-related macular degeneration. Retina 31(1):26-30

13. Toalster N, Russell M, Paul N (2013) A 12-month prospective trial of inject and extend regimen for ranibizumab treatment of agerelated macular degeneration. Retina 33(7):1351-1358

14. Berg K, Pedersen TR, Sandvik L, Bragadóttir R (2015) Comparison of ranibizumab and bevacizumab for neovascular age-related macular degeneration according to LUCAS treat-and-extend protocol. Ophthalmology 122(1):146-152

15. Spaide R (2007) Ranibizumab according to need: a treatment for agerelated macular degeneration. Am J Ophthalmol 143(4):679-680

16. AAO Retina/Vitreous PPP Panel, Hoskins Center for Quality Eye Care (2015) Age-related macular degeneration PPP - updated 2015, Preferred Practice Pattern Guideline, American Academy of Opthalmology

17. Hatz K, Prunte C (2017) Treat and extend versus pro re nata regimens of ranibizumab in neovascular age-related macular degeneration: a comparative 12 month study. Acta Ophthalmol 95(1):e67e72. https://doi.org/10.1111/aos.13031

Publisher's note Springer Nature remains neutral with regard to jurisdictional claims in published maps and institutional affiliations. 